Document downloaded from:

http://hdl.handle.net/10251/115613

This paper must be cited as:

Rubio Michavila, C.; Minin, IV.; Minin, OV.; Tarrazó-Serrano, D.; Castiñeira Ibáñez, S. (2019). Tunable depth of focus of acoustical pupil masked Soret Zone Plate. Sensors and Actuators A Physical. 286:183-187. doi:10.1016/j.sna.2018.11.053

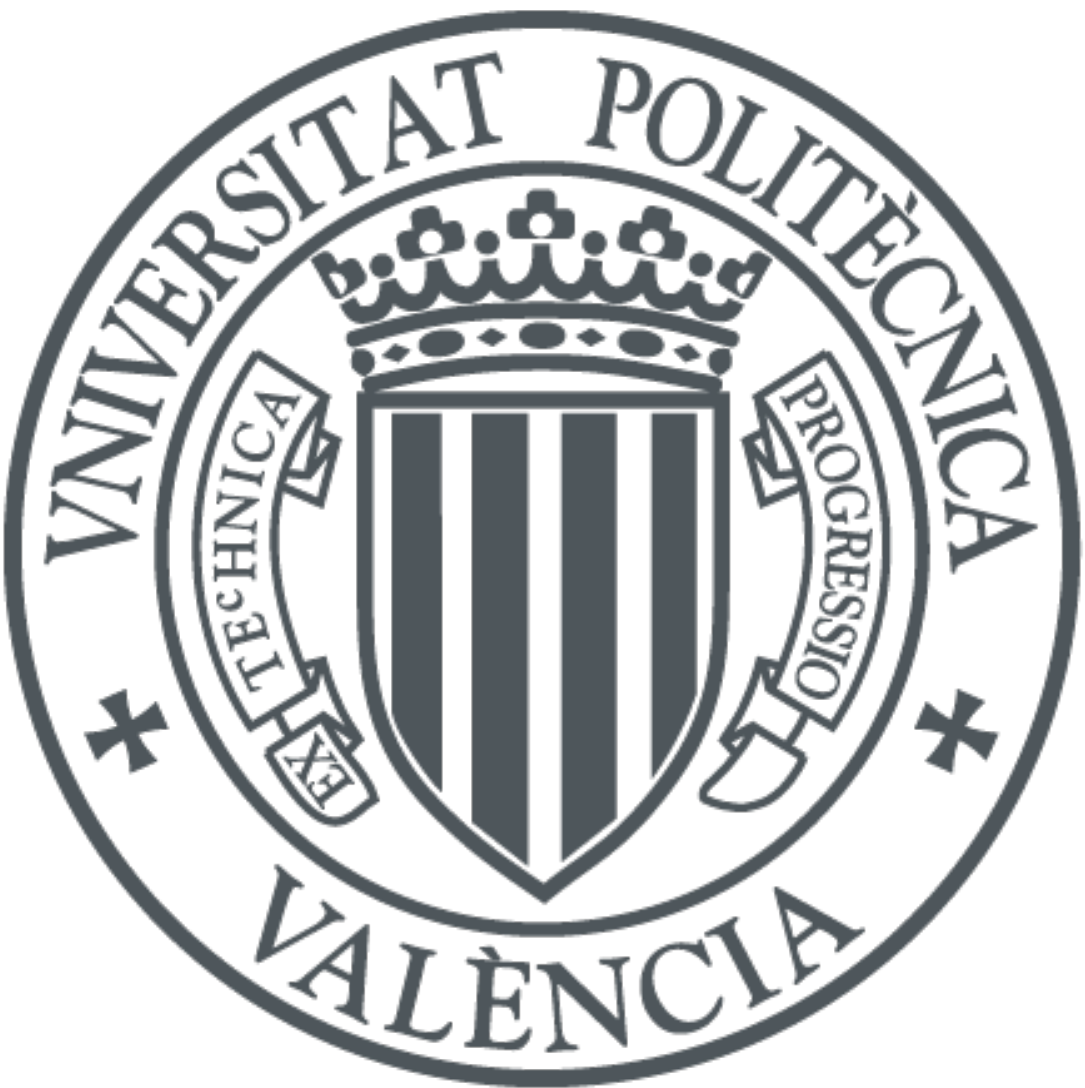

The final publication is available at

https://doi.org/10.1016/j.sna.2018.11.053

Copyright Elsevier

Additional Information 


\title{
Tunable depth of focus of acoustical pupil masked Soret Zone Plate
}

\author{
Sergio Castiñeira-Ibáñez ${ }^{\mathrm{a}}$, Daniel Tarrazó-Serrano ${ }^{\mathrm{a}}$, Oleg V. Minin ${ }^{\mathrm{b}}$, Constanza Rubio ${ }^{\mathrm{a}, *}$, Igor V. Minin ${ }^{\mathrm{c}, *}$ \\ ${ }^{a}$ Centro de Tecnologías Fúsicas, Universitat Politècnica de València, Camí de Vera s/n, 46022, València, Spain. \\ ${ }^{b}$ Tomsk State University, 30 Lenin Avenue, Tomsk, 634050, Russia \\ ${ }^{c}$ Tomsk Politechnical University, 36 Lenin Avenue, Tomsk, 634050, Russia
}

\begin{abstract}
In acoustical lenses both resolution and depth of focus are determined by diffraction and the smaller the lens aperture the worse the resolution and the greater the depth of focus. Diffraction-limited resolution has a Soret Zone Plate, but a long depth of focus has an axicon. Nevertheless, these are two different devices each of which requires its own independent design. In this paper we have shown that the transition from focusing to a diffraction limited spot to a quasi-diffraction free beam can be realized in the same focusing device without changing its topology. It has been shown that using a classical planar Soret Zone Plate lens made of a concentric array of circular aperiodical rings with an amplitude pupil mask placed closely to the surface of lens allows to form a quasi-Bessel beams under specific conditions, part of a diffracted wave collimates, producing an elongated focus. Experiments are performed in water tanks in order to verify the simulation results. Experimental verification shown that the depth of focus of a pupil-masked Soret Zone Plate increases 1.63 times and resolution increases 1.2 times (with minimal beam waist about of 0.67 of wavelength and depth of focus about 5.72 of wavelength). By dynamically controlling the size of the amplitude pupil mask, it is possible to quickly control the depth of focus of an acoustic lens.
\end{abstract}

Keywords: acoustic bessel beam, axicon, ultrasonic lens

\section{Introduction}

Different ways for focusing and modulation of wavefronts have been researched by scientist and engineers because of its possibilities and applications in different physic

5 areas. The applications of ultrasonic sensors are very varied ranging from their use to verify trees in poor condition, being a non-invasive technique [1, even to produce microfluidic movement by means of slightly focused acoustic waves [2]. Zone Plates are one of these devices based 10 on both refraction and diffraction mechanisms which can ${ }_{35}$ achieve wave focusing.

Zone Plates, which consist of alternating opaque and transparent Soret Zone Plates (SZP) or phase reversal Fresnel Zone Plates (FZP) rings [3, 4, 5, 6, are based on 15 the diffraction phenomenon to modulate and focus acoustic waves, and as a simple example of acoustic lens has a 40 long history [7, 8, 9] Acoustic Zone Plates have been used in different applications due to their flat surface, dimension and fabrication advantages [10, 11, 12]. For example, self-focused acoustic ejectors using the double Fresnel Zone Plate were investigated 13. SZP for underwater ultrasounds alternating opaque and transparent zones made of soft silicone rubber was developed [14, 15]. Results had ${ }_{45}$ shown a main lobe widening but also, a small reduction

\footnotetext{
${ }^{*}$ Corresponding authors:

Email addresses: crubiom@fis.upv.es (Constanza Rubio), prof.minin@gmail.com (Igor V. Minin)
}

25 of the first Side Lobe Level (SLL). Zone plate with fractal structures was studied [16]. Acoustic Zone Plate lenses with extraordinary transmission based on Fabry-Perot resonances were investigated [17. Modification of Zone Plate, which operates with piston emitters, was studied [18].

However, relative small Depth of Focus $(D o F)$ is one of the problems that SZP lenses have. To increase the depth of focus and spatial resolution many papers investigated so-called acoustic Bessel beams due to the central core spot size of the Bessel beam, which is defined from the properties of the zeros of a Bessel function. For example, with the help of an axicon, it is possible to form a Bessel beam of zero order [19, whose central spot diameter at the halfwave intensity (Full Width at Half Maximum, FWHM) is equal to:

$$
F W H M_{a}=0,36 \lambda / N A
$$

The resulting focusing spot will be about $30 \%$ smaller than the size of the Airy disk, formed by a lens with the same numerical aperture (NA):

$$
F W H M_{l}=0,51 \lambda / N A
$$

Stratton originally proposed these Bessel beams in 1941 20. Acoustic Bessel beams have been excited using refractive acoustical axicons 21, 22, in analogy to the optical case. Bessel beams of sound waves were also reported [23. 24] and have been studied for realization with two50 dimensional acoustic arrays 25. In contrast to refractive 
axicon, diffractive flat axicon 26 has the same advantages as a flat Soret or Fresnel Zone Plates.

For example, a flat acoustic lens with aperiodical structure to transform a divergent beam into a Bessel like beam 55 was reported [27]. However, the most convenient way to form acoustic Bessel beams is by using annular transducer arrays [25, 28]. Recently, the acoustic Bessel-like beam by means of an axisymmetric periodical grating of rigid tori was also reported 29, but it could be mentioned if Babi60 net's principle 30] is considered, both approaches (SZP and rigid tori scatterers) are considered equivalent. Thus, today acoustic diffraction flat lenses and axicon are successfully used in different applications. Nevertheless, these are two different devices each of which requires its own independent design.

In this paper, we propose and demonstrate that the transition from focusing to a diffraction-limited spot (lens with aperiodical structure) to a quasi-diffraction-free beam (axicon with periodical structure) can be realized in the 70 same focusing device without changing its topology. We show a technique for acoustic Bessel-like beam formation using a classical planar Soret Zone Plate lens made of concentric array of circular aperiodical rings with amplitude pupil mask placed closely the surface of SZP. We show 75 that, under specific conditions, part of a diffracted wave collimates, producing an elongated focus. Numerical calculations using Finite Element Method (FEM) of acoustic waves propagating through such a lens were used to observe the complete acoustic field. Finally, the experimental verification of quasi-Bessel beam formation by two type of SZPs is reported.

\section{Methodology}

To simplify the problem without loss of generality, geometrical optics interpretation is considered (Fig. 1).

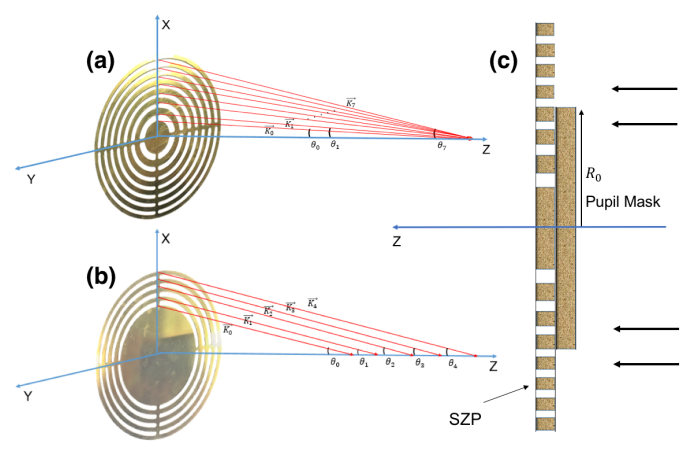

Figure 1: Ray-tracing for SZP (a) and PSZP (b). Combination of pupil-mask and SZP (c).

As it well-known binary SZP lens consists of concentric ring, which can be treated as a quasi-periodic grat-125 ing with different local grating constants at different radii. For point focusing, the normal incident wave is diffracted

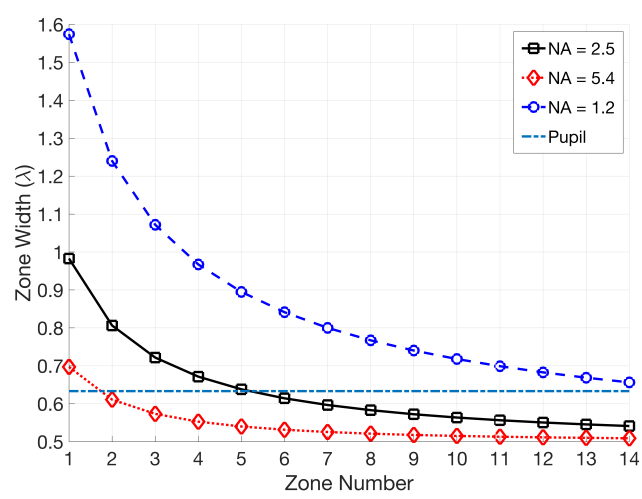

Figure 2: Zone width vs. number of transparent zones for different NA varying of focal length. Dash-dotted line is located at the value where the difference of zone width is less than $\lambda / 5$.

towards the designed focal point by these local gratings. The diffraction angles change in order to ensure the formation of a focal spot (Fig. 117). In this content SZP with pupil mask (PSZP) may be also considered as ring gratings because of the widths of transparent zones are almost constant while the pupil is blocking a central part of SZP. Thus, the local gratings diffract the incident waves towards different points on the optical axis with a small range of diffraction angle (Fig. 1b).

For axicon 26] due to the diffraction angle $(\theta)$ of the ring grating and the grating period $(P)$ are related by the Bragg's law: $31 P \cdot \sin (\theta)=m \lambda$, where $m$ is the diffraction order and $\lambda$ is the incident wavelength of acoustic wave, it produces Bessel beam. In the case of SZP with pupil mask, whose local periods $\mathrm{P}_{\mathrm{i}}$ are not regular, we block those rings that can not be considered as periodic, and we may write that local $\mathrm{P}_{\mathrm{i}}=\Delta \mathrm{r}_{\mathrm{i}}$, where $\Delta \mathrm{r}_{\mathrm{i}}$ is a width of i-th transparent zone and due to $\Delta r_{i}$ is almost constant, and thus diffraction angles $\left(\theta_{\mathrm{i}}\right)$ also almost constant, such pupil-masked SZP also will produce a quasi-Bessel beams.

When choosing the size of the pupil mask there is a compromise. On the one hand, the period and size of the slits should be as close to the periodic as possible. On the other hand, it is necessary to leave as many zones as possible on a fixed diameter of the lens in order to provide a sufficient length of focus. We settled on the criterion when $\Delta r_{i}$ ) is less than the $\lambda / 5$ (Fig. 2)

To verify the above-mentioned idea and simulate the acoustic pressure distribution in axisymmetric models of SZP we used the commercial software COMSOL Multisics with FEM method 32 . Underwater transmission is considered with typical water values of sound speed propagation $(\mathrm{v}=1500 \mathrm{~m} / \mathrm{s})$ and density $\left(\rho=1000 \mathrm{~kg} / \mathrm{m}^{3}\right)$. Design frequency (f) is $250 \mathrm{kHz}$, therefore wavelength $(\lambda)$ is $6 \mathrm{~mm}$. Lenses were defined full rigid considering Neumann condition. Mesh geometry was selected in triangles with minimum element size of $\lambda / 14$ and maximum element size of $\lambda / 8$ to prevent numerical dispersion. 
(a)

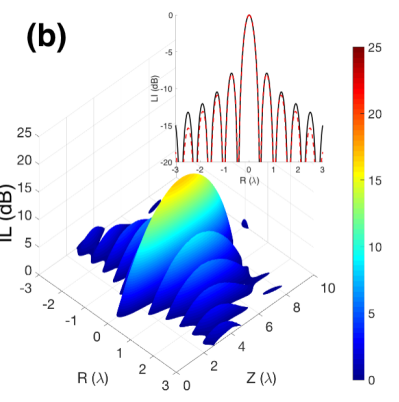

Figure 3: Acoustic intensity distributions near the focus of SZP (a) and PSZP (b). Inset in (b) shows axial comparison between $\mathrm{J}_{0}{ }^{2}$ (black) and pupil mask (red dashed line).

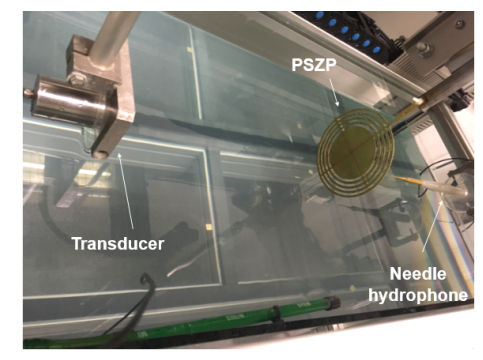

Figure 4: Experimental set-up. Assembly placed in a water tank for 165 using the ultrasonic immersion transmission technique.

The SZP lenses with high $\mathrm{NA}=2.5$ was selected (the $\mathrm{NA}$ is determined by both the diameter of the lens and the specified primary focal length as $\mathrm{NA}=\mathrm{D} / 2 \mathrm{~F}$ ) due to ${ }^{170}$ focusing of this device with $\mathrm{F}=4.5 \lambda$ leads to a very compact beam with a short working distance. It could be noted that we can't use the well-known equations for NA due to small focal distance where the paraxial approximaare not valid. Analysis of the simulations shown that the focused field in the cases of PSZP (Fig. 3p) instead of classical SZP (Fig. 3a) has a characteristic structure for quasi-Bessel beams with intensity profiles that closely resemble the ideal $J_{0}^{2}$ transverse-intensity distribution of Bessel beams (see the inset in Fig. 3b). FWHMs for these acoustical lenses are as follows: SZP $0.84 \lambda$, PSZP $0.64 \lambda$, Bessel beams $0.64 \lambda$. Accordingly, the DoFs are: for SZP $2.84 \lambda$ and for PSZP $5.94 \lambda$.

\section{Experimental Results}

In order to carry out the experimental measurements, ${ }^{185}$ the SZPs have been implemented in brass using a mechanization process. The material selected for its construction is brass because it has a relatively low transmission coefficient and behaves as an opaque sound material as shown in the Fig. 4. All the measures have been done using an automated full precision measurement system in order to validate and compare the proposed lenses. This system consists of a fixed ultrasonic transducer and a 3D positioned hydrophone 33] as shown in the Fig. 4. This system (a)
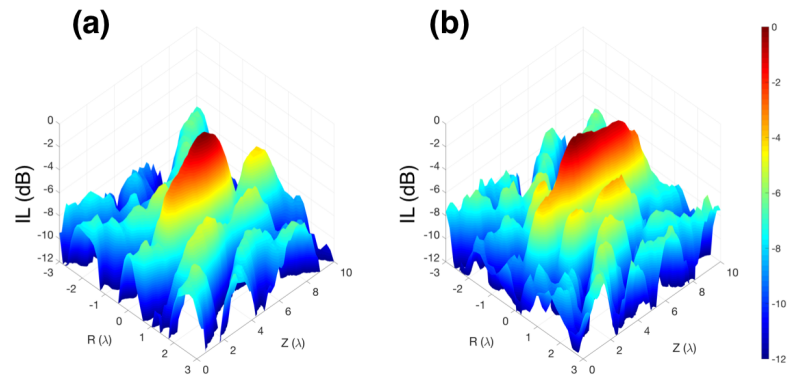

Figure 5: Experimental normalized by the maximum intensity 3D planes for (a) SZP and (b) PSZP.

The transducer used is a $250 \mathrm{kHz}$ Imasonic piston with $32 \mathrm{~mm}$ of active diameter and the needle hydrophone is a MPM1/1 from Precision Acoustic Ltd. made of polyvinylidene fluoride with a diameter of $1.5 \mathrm{~mm}$. To ensure the far field condition, the distance between the transducer and the Zone Plate Lens was $0.4 \mathrm{~m}$. Flat transfer function between 0.2 and $15 \mathrm{MHz}$ provides accuracy on the measurements. Fig. 5 shows the normalized by the maximum intensity planes for SZP and PSZP respectively. Experimental results are in good agreement with the numerical predictions. As it seen from the Fig. 5 b, the consideration of a pupil mask generates a distribution of energy along the longitudinal axis narrowing the beam and generating a quasi-Bessel beam distribution. Analysis of simulations and experimental investigations show that by adding an amplitude pupil mask shielding the central part of the lens, the focusing properties of the Soret lens dramatically change the field structure in the focusing region becomes close to the Bessel beam. Experimental verification confirm that the depth of focus increase to 1.63 times and resolution increase to 1.2 times (with minimal beam waist about of $0.67 \lambda$ and depth of focus about $5.72 \lambda$ ). It could be noted that in optical band central pupil mask method was used to block the light through the central part in the point-focusing super-oscillatory lenses to achieve a $D o F$ more than $5 \lambda$; however, the realization of such an extended DoF was at the expense of degradation in the focus transverse size [34, 35.

\section{Conclusion}

In this paper, it is shown that the focusing properties of an acoustic binary Zone Plate can be controlled without changing the structure of the zones by introducing an amplitude pupil mask. In this case, diffraction by a quasiperiodic ring lattice formed by peripheral Fresnel zones leads to the formation of an elongated focus corresponding to the structure of a quasi-Bessel beam. The depth of focus is determined by both the size of the pupil mask and the diameter of the acoustic lens. Therefore, circular gratings based on pupil-masked SZP, will enable an intense quasi-Bessel beam with diffraction limit waist and much 
extended DoF. By dynamically changing the size of the amplitude pupil mask, it is possible to quickly control the depth of focus of the acoustic Soret lens.

Experimental verification shows that the depth of field $\mathrm{d}_{260}$ ncrease to 1.63 times and resolution increase to 1.2 times (with minimal beam waist about of $0.67 \lambda$ and depth of focus about $5.72 \lambda$ ). Such systems can find wide application in nondestructive devices, acoustic microscopy, imaging ${ }_{265}$ systems and control of nano and micro particles. The extremely easy generation of these beams using pupil binary mask proposed here can handle high wave powers, thus is particularly suitable for applications in plasma chan-270 nel generation, nonlinear optics, and x-ray or microwave beam manipulation. Moreover, this approach can also be directly applied to light and matter waves.

\section{Acknowledgments}

This work has been supported by Spanish MINECO (TEC2015-70939-R) and was partially supported by Tomsk ${ }^{280}$ Polytechnic University Competitiveness Enhancement Program.

\section{References}

[1] C. L. Goh, R. A. Rahim, M. H. F. Rahiman, M. T. M. Talib, Z. C. Tee, Sensing wood decay in standing trees: A review, Sensors and Actuators A: Physica doi:10.1016/j.sna.2017. 90 11.038

[2] X. Zhu, E. S. Kim, Microfluidic motion generation with acoustic waves, Sensors and Actuators A: Physical 66 (1-3) (1998) 355360. doi:10.1016/S0924-4247(97)01712-3

[3] L. Rayleigh, Scientific Papers by John William Strutt, Baron 295 Rayleigh, Vol. 3 (1902).

[4] J. L. Soret, Ueber die durch kreisgitter erzeugten diffractionsphänomene, Annalen der Physik 232 (9) (1875) 99-113.

[5] R. W. Wood, LIII Phase-reversal zone-plates, and diffractiontelescopes, The London, Edinburgh, and Dublin Philosophical 300 Magazine and Journal of Science 45 (277) (1898) 511-522.

[6] A. J. Fresnel, Calcul de l'intensite de la lumiere au centre de l'ombre d'un ecran et d'une ouverture circulaires eclairepar un point radieux, SPIE MILESTONE SERIES MS 128 (1996) 310 .

[7] K. Yamada, H. Shimizu, New types of ultrasound focusing transducer sysmtems, Japanese Journal of Applied Physics 21 (S3) (1982) 135.

[8] K. Yamada, H. Shimizu, Planar-structure focusing lens for acoustic microscope, Journal of the Acoustical Society of Japan 310 (E) 12 (3) (1991) 123-129. doi:10.1250/ast.12.123

[9] S. A. Farnow, B. A. Auld, Acoustic fresnel zone plate transducers, Applied Physics Letters 25 (12) (1974) 681-682. doi: $10.1063 / 1.1655359$

[10] E. Schonbrun, C. Rinzler, K. B. Crozier, Microfabricated water ${ }_{315}$ immersion zone plate optical tweezer, Applied Physics Letters 92 (7) (2008) 071112. doi:10.1063/1.2837538

[11] I. Amemiya, H. Yagi, K. Mori, N. Yamamoto, S. Saitoh, C. Tanuma, S. Hirahara, Ink jet printing with focused ultrasonic beams, in: Proc. NIP 13: International Conference on 320 Digital Printing Technologies, 1997, pp. 698-702.

[12] I. V. Minin, O. V. Minin, 3D High-Quality Ultrasonic Imaging, InTech, 2011. doi:10.5772/16460

[13] S. F. Hon, K. W. Kwok, H. L. Li, H. Y. Ng, Self-focused acoustic ejectors for viscous liquids, Review of scientific instruments 325 81 (6) (2010) 065102. doi:10.1063/1.3442526
14] D. C. Calvo, A. L. Thangawng, M. Nicholas, C. N. Layman, Thin fresnel zone plate lenses for underwater acoustics: Modeling and experiments, in: OCEANS'15 MTS/IEEE Washington, IEEE, 2015, pp. 1-6.

[15] D. C. Calvo, A. L. Thangawng, M. Nicholas, C. N. Layman, Thin fresnel zone plate lenses for focusing underwater sound, Applied Physics Letters 107 (1) (2015) 014103. doi:10.1063/ 1.4926607

[16] S. Castiñeira-Ibáñez, D. Tarrazó-Serrano, C. Rubio, P. Candelas, A. Uris, An ultrasonic lens design based on prefractal structures, Symmetry 8 (4) (2016) 28. doi:10.3390/sym8040028.

[17] M. Molerón, M. Serra-Garcia, C. Daraio, Acoustic fresnel lenses with extraordinary transmission, Applied Physics Letters 105 (11) (2014) 114109. doi:10.1063/1.4896276.

[18] S. Pérez-López, J. M. Fuster, P. Candelas, C. Rubio, F. Belmar, On the use of phase correction rings on fresnel zone plates with ultrasound piston emitters, Applied Physics Letters 112 (26) (2018) 264102. doi:10.1063/1.5036712

[19] V. P. Kalosha, I. Golub, Toward the subdiffraction focusing limit of optical superresolution, Optics letters 32 (24) (2007) 3540-3542. doi:10.1364/0L.32.003540.

[20] J. A. Stratton, Electromagnetic Theory, McGraw-Hill, 1941.

[21] C. B. Burckhardt, H. Hoffmann, P. A. Grandchamp, Ultrasound axicon: A device for focusing over a large depth, The Journal of the Acoustical Society of America 54 (6) (1973) 1628-1630. doi:10.1121/1.1914459

[22] S. Nagai, K. Iizuka, A practical ultrasound axicon for nondestructive testing, Ultrasonics 20 (6) (1982) 265-270. doi: 10.1016/0041-624X(82) 90047-6

[23] D. K. Hsu, F. J. Margetan, D. O. Thompson, Bessel beam ultrasonic transducer: Fabrication method and experimental results, Applied physics letters 55 (20) (1989) 2066-2068. doi: 10.1063/1.102107

[24] P. Katchadjian, C. Desimone, A. D. Garcia, Application of axicon lenses in ultrasonic techniques, in: AIP Conference Proceedings, Vol. 1211, AIP, 2010, pp. 1043-1050.

[25] J. Y. Lu, J. F. Greenleaf, A study of two-dimensional array transducers for limited diffraction beams, IEEE transactions on ultrasonics, ferroelectrics, and frequency control 41 (5) (1994) 724-739. doi:10.1109/58.308509

[26] J. Dyson, Circular and spiral diffraction gratings, Proc. R. Soc. Lond. A 248 (1252) (1958) 93-106. doi:10.1098/rspa.1958. 0231

[27] Z. Xu, W. Xu, M. Qian, Q. Cheng, X. Liu, A flat acoustic lens to generate a bessel-like beam, Ultrasonics 80 (2017) 66-71. doi:10.1016/j.ultras.2017.05.005

[28] H. Masuyama, K. Mizutani, Acoustic beam scanning using annular transducer array introducing decentering operation, JSME International Journal Series C Mechanical Systems, Maㅁ chine Elements and Manufacturing 49 (3) (2006) 681-686. doi: $10.1299 /$ jsmec.49.681

[29] N. Jiménez, V. Romero-García, R. Picó, A. Cebrecos, V. J. Sánchez-Morcillo, L. M. Garcia-Raffi, J. V. Sánchez-Pérez, K. Staliunas, Acoustic bessel-like beam formation by an axisymmetric grating, EPL (Europhysics Letters) 106 (2) (2014) 24005. doi: 10.1209/0295-5075/106/24005

[30] J. Babinet, Memoires d'optique méteorologique, 1837.

[31] W. H. Bragg, W. L. Bragg, et al., The reflection of x-rays by crystals, Proc. R. Soc. Lond. A 88 (605) (1913) 428-438. doi: 10.1098/rspa.1913.0040

[32] COMSOL-Multiphysics, COMSOL Multiphysics User Guide (version 4.3a) (2012).

[33] C. Rubio, J. M. Fuster, S. Castiñeira-Ibáñez, A. Uris, F. Belmar, P. Candelas, Pinhole zone plate lens for ultrasound focusing, Sensors 17 (7) (2017) 1690. doi:10.3390/s17071690

[34] T. Roy, E. T. F. Rogers, G. Yuan, N. I. Zheludev, Point spread function of the optical needle super-oscillatory lens, Applied Physics Letters 104 (23) (2014) 231109. doi:10.1063/1. 4882246

[35] F. Qin, K. Huang, J. Wu, J. Jiao, X. Luo, C. Qiu, M. Hong, Shaping a subwavelength needle with ultra-long focal length by 
focusing azimuthally polarized light, Scientific reports 5 (2015) 9977. doi:10.1038/srep09977 